\title{
Removing Unwanted Objects from an Image using Image in-painting
}

\author{
Manjushri D. Sarode \\ Department of Computer Engineering, \\ MMCOE,Pune \\ SavitribaiPhule Pune \\ University,Pune, India.
}

\author{
Rahul Dagade \\ Department of Computer Engineering, \\ MMCOE,Pune \\ SavitribaiPhule Pune \\ University,Pune, India.
}

\begin{abstract}
Image in-painting refers to a technique used for refill the missing regions of an imageand recover the corrupted image.Image in-painting or completion is a technique to restore a damaged image completely. In-painting approaches play a important role in numerous applications like object deletion, scratch deletion, Image restoration etc. Two classes of algorithms addressed this problem, these classes are: (i)Texture synthesis algorithms for creating large image regions from sample textures, and (ii) In-painting techniques for filling in small image region or gap. Various strategies have been proposed different Exemplar-based image inpainting algorithms to recover the structure of damaged images. This paper introduces a novel and efficient exemplar based Image In-painting Algorithm with investigating natural image patches. It contain in-painting techniques with respect to restoration of image. The goal of any image in-painting algorithm is to reconstruct the missing or damaged regions.
\end{abstract}

\section{Keywords}

In-painting, super-resolution, Image Processing, Exemplarbased in-painting.

\section{INTRODUCTION}

Image processing is one of the important forms of signal processing in which the input is an image, such as a photograph. The output of image processing may be either an image or a set of parameters or characteristics related to the image. Because of the wide application of digital cameras and the digitalization of old photos, in-painting has become one of the most important process, which is to be operated on digital images. More than scratch removing, object removal and textual removal can be carried out using in-painting techniques. Furthermore, in-painting can also be applied in applications like super resolution and image compression.

Image in-painting is the art of filling the missing region in an image. The main purpose of in-painting is to recover or reconstruct missing region in a manner so that it seems reasonable to the human eye. There are several approaches proposed for the same. In the digital world, in-painting (Image Interpolation)are refer to the application of sophisticated algorithms to replace lost, corrupted or damaged part of the image data (mainly small region or to remove small defects).

The important goal of super resolution is to create a high resolution image from one or more low resolution input images. Super resolution methods can be divided into two parts: 1. Single image super resolution 2. Classical multiimage super resolution, in classical image super resolution it takes same scene from set of low resolution images. Each low resolution image imposes a set of linear constraint on the unknown high resolution intensity values. If the images that are in low resolution are available then the set of equations are determined and can be solved to create one of the high resolution images.

Image In-painting is defined as a procedure to fill the missing regions or area in an image. Existing Diffusion based method uses partial equations and variation methods for In-painting. But when the hole that is going to be filled is large, the resultant image using diffusion based method lead to introduce blur in the image. Due to high computational time and problems when the hole that is going to be filled is large there contain many difficulties in In-painting algorithms. Recently research on this field has been very active, started by numerous applications: object removal in a context of editing, loss concealment in the form of impaired image transmission,restoring image from scratches or text overlays, the view point are different from those images that re captured by cameras when dis-occulation in image based rendering(IBR).

Existing method can be classified into two categories:

\subsection{Diffusion-based method}

Diffusion based method use various methods and partial equations. In this method when the hole that is going to be filled is large, the resultant image of diffusion based method lead to introduce blur in the image. It gives the better result when the hole to be filled is very small. Diffusion-based method uses variation theory.

\subsection{Exemplar-based method}

The Exemplar-based method copies the best match patches from the image. This technique is very cheap and it generates the new texture by copying the color from the source value. There are many difficulties in In-painting algorithms due to high computational time and problems when the hole that is going to be filled is large. By using hierarchical approach first will going to convert image into low resolution (LR) image, the super resolution (SR) method is used only when the inpainting method is applied on low resolution of input image.and then use K-NN(K-Nearest Neighbor)algorithm for in-painting. After In-painting to improve the quality of image will use super resolution algorithm to improve quality of image.

\section{LITERATURE REVIEW 2.1 FaouziBenzarti, Hamid Amiri[1]}

In this paper, author has been proposed system for in-painting using Diffusion based In-painting technique. Diffusion based In-painting was the first digital In-painting approach. In diffusion based technique firstly missing area is going to filled by diffusing image information from the well known region into the missing region at pixel level. These algorithms are based on theory of Partial Differential (PDE) variation method. The diffusion- based In-painting algorithm produces poor results or filling the non-textured or relatively smaller 
missing region. The main drawback of the diffusion process is it introduces some blur image, which becomes noticeable when filling region is larger. All the PDE based in painting models are more suitable for completing small, non-textured targeted region.

\subsection{Jason C.Hung,chun-Hong Hwang,Yi-Chun Liao,NickC,Tang and Ta-Chen[3]}

In this paper,author expands an exemplar based image inpainting by integrating by Bezier curve. Bezier curve is use to build missing edge information.The foundation of this technique is the contour lines restoring and exemplar based image in-painting technique.In this approach firstly,make use of mean shift segmentation for understanding color segmentation in damaged photograph/image.The Bezier curve is utilized to join the missing contour lines to rebuild main structure in damaged area but this technique require more time to perform.

\subsection{Bertalmio, L.Vese, G. SapiroAnd S. Ocher [3]}

Have proposed PDE based algorithm .PDE based algorithm is use to continue photometric information and geometric that arrives at the border of the occluded area into area itself. This can be done by combining information in form of minimal change using isophote lines. Disadvantages -This algorithm would not produce good results if damaged regions are large one. If the missing regions are large this algorithm will take so long time to perform and it will not produce good results. This method neither connects broken edges/linenor do greats texture patterns. PDE based algorithms were focused on maintaining the structure of the In-painting area and hence resulting image of these algorithms are blurred.

\subsection{ShivaniGaikar And NehaKhairnar[4]}

In this paper, author eliminated the unnecessary objects or data from the original photographs/image but the modification is notobserved by user.This will made by considering the three algorithms that is: Exemplar Based Image In-painting Technique, DCT (Discrete Cosine Transform)Based Technique and FMM(Fast Matching Method). ExemplarBased Image In-painting Technique, DCT Based Technique which efficiently and economically produce new texture by sampling and copying color value from source. The Fast Matching Method is utilizing to eliminate all scratches within photograph/image. Disadvantage of this technique is Implementation of such system is difficult.

\subsection{MonaliDesai[5]}

In this paper,author presents an adapted fast and improved exemplar based image in-painting technique to solve the unknown row filling difficulties. This modified technique is adaptation in updating criteria in fast and enhanced exemplar based image in-painting. It resolves difficulty of unknown row filling and provides results. But the resultant image produced by this approach is still suffer from some difficulties due to which it adds few unwanted incorrect information from background in the photograph/image.

\subsection{SheetalBadgujar and N.M. Shahane[6]}

In this paper author enhanced the performance of exemplar based image in-painting algorithmby introducing two different techniques.Both techniques utilize patch propagation by internally propagating the image patches from the source area into interior of the target area patch by patch. In the first technique errors often arise when little number of well-known pixels is used to represent a big unknown area. The patch shifting technique offers more significant target patch than traditional exemplar based technique.

\subsection{Efros, A.A., Leung, T.K. [7]}

Have proposed texture synthesis by non-parametric sampling Texture synthesis based in-painting algorithms are one of the earliest methods of image In-painting and these algorithms are used to complete the missing regions by using similar neighborhoods of the damaged pixels. This algorithm synthesize new image pixels from origin pixel. and then perform operation on image.For e.g., Markov Random Field (MRF) is basically used to model the local distribution of the pixels and new texture is synthesized by finding all similar neighborhoods and querying existing texture. So their differences exist mainly in how continuity is maintained between In-painting holeand existing pixels. The main objective of texture synthesis based In-painting is to generate texture patterns, which is similar to a given sample pattern, in such a way that it reproduced texture retains the statistical properties of its origin/root texture.

\subsection{AntoSilviya and V.R.Bhuma [8]:}

In this paper author enhanced the performance of exemplar based image in-painting by introducing new technique with spatiogram. A spatiogram is a photograph/image descriptor. Spatiogram is mixture of histogram with mean and covariance of the location with each other. The unnecessary area removed by means of associated neighborhood technique and completion of missing area is made by Laplacian approximation technique. Image is subdivided into small blacks based on the size of hole. The quality of image can be improved by using histogram equations, this technique require more time to implement.

\subsection{Criminisi, P. P'erez and K. Toyama[9]}

Have proposed a system with Texture Synthesis algorithm. The limitation of this paper is that the synthesis of regions for which similar patches do not exist does not produce reasonable results; the algorithm is not designed to handle curved structures. This algorithm does not handle depth ambiguities.

Table 1.Comparison of various image in painting methods

\begin{tabular}{|l|l|l|}
\hline Method & Advantages & Disadvantages \\
\hline $\begin{array}{l}\text { Differential } \\
\text { Equation }\end{array}$ & $\begin{array}{l}\text { It produces good } \\
\text { results if missing } \\
\text { regions are small } \\
\text { one. }\end{array}$ & $\begin{array}{l}\text { If missing regions } \\
\text { are large algorithm } \\
\text { takes long time } \\
\text { and does not } \\
\text { produce good } \\
\text { results. }\end{array}$ \\
\hline $\begin{array}{l}\text { Exemplar Based } \\
\text { In painting }\end{array}$ & $\begin{array}{l}\text { Efficient for large } \\
\text { target regions. }\end{array}$ & $\begin{array}{l}\text { Calculation time is } \\
\text { more. }\end{array}$ \\
\hline $\begin{array}{l}\text { Texture } \\
\text { Innthesis Based }\end{array}$ & $\begin{array}{l}\text { Performs well in } \\
\text { approximating } \\
\text { textures. }\end{array}$ & $\begin{array}{l}\text { Difficulty in } \\
\text { handling natural } \\
\text { images. Not } \\
\text { suitable for a large } \\
\text { objects }\end{array}$ \\
\hline $\begin{array}{l}\text { Wavelet } \\
\text { Transform Based }\end{array}$ & $\begin{array}{l}\text { Good image } \\
\text { representation } \\
\text { quality. Maintain } \\
\text { texture and } \\
\text { structure quality. }\end{array}$ & $\begin{array}{l}\text { Difficulty for large } \\
\text { regions and mask } \\
\text { for regions are } \\
\text { defined manually. }\end{array}$ \\
\hline $\begin{array}{l}\text { Semi-Automatic } \\
\text { and Fast In } \\
\text { painting }\end{array}$ & $\begin{array}{l}\text { Computational time } \\
\text { is less }\end{array}$ & $\begin{array}{l}\text { This technique is } \\
\text { not suitable in } \\
\text { filling large hole } \\
\text { regions. Results in } \\
\text { blur effect in } \\
\text { image. }\end{array}$ \\
\hline
\end{tabular}




\section{IMPLEMENTATION DETAILS}

\subsection{Work Breakdown Structure}

Removing Unwanted Objects From An Image Using Image In painting

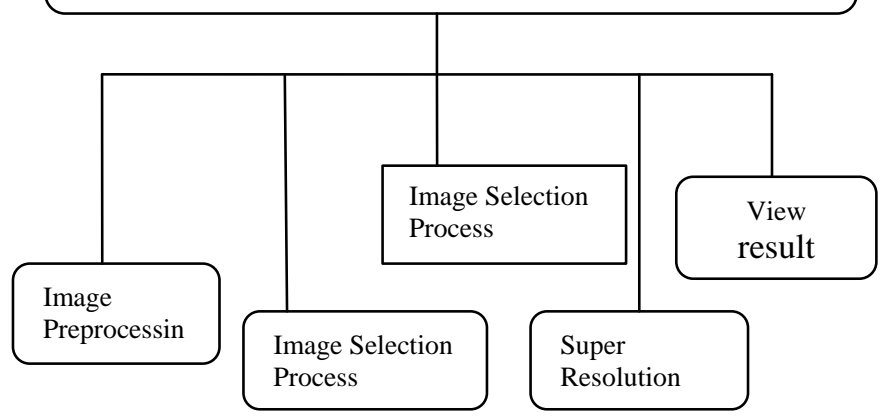

Fig.1 Work Breakdown structure

The breakdown structure mainly focuses on following operations:

Module 3.1.1: Image Preprocessing

Module 3.1.2: Image Selection Process

Module 3.1.3: In-painting Method

Module 3.1.4: Super Resolution

Module 3.1.5: View result

Module 3.1.1: Image Preprocessing

The selected image should be processed bye using image processing module. If user selected low resolution image then it can be directly forwarded to In-painting method but if user selected high resolution image then it should first converted into low resolution image and then forwarded to In-painting.

Module 3.1.2: Image Selection Process

User must have to select the area on input image whereuser needs to do in-painting. All the parameters of the images are going to calculate and stores in a file. User is going to select area where he wants to do in-painting. Based upon the operation apply in-painting algorithm on the area of image which user need to in-paint.

\section{Module 3.1.3: In-painting Method}

After selection of the object that selected object must remove from image, then using in painting algorithm the nearest patches should select to form the complete image..

Following are the steps to be followed in the in-painting process:

1.Patch priority and filling order

\section{Texture synthesis}

Module 3.1.4:Super-resolution

After In-painting Process completion the in painted image should convert into high resolution image this can be done by using super resolution method. Low resolution image can be converted into high resolution image using super resolution algorithm. Super-resolution algorithm is described in 5 steps:

\subsubsection{Dictionary building:}

Dictionary building is corresponds between low and high resolution image patches. The size of dictionary is specified by the user which may affect the overall speed or quality.

\subsubsection{Filling order of HR picture:}

Filling the holes in image is depend on the priority if patches in image. As compared to raster-scan filling order this method improves the quality of in-painted image.

\subsubsection{Similarity Computation:}

For the similar patches computation K-NN algorithm is used.

\subsubsection{Weight Computation:}

The similarity distance is used to compute the weight.

\subsubsection{Stitching:}

The stitching algorithm is only used when all pixel values in the overlapping region are known or already synthesized.

\section{PROPOSED SYSTEM}

Propose framework for removing the unwanted object from images and refill the object space using image in-painting.

\subsection{Input}

The image is taken as input of the system that can be of any size and format.To remove unwanted object from it.

\subsection{ROI generation}

A region of interest (ROI) is a selected subset of samples (selected area) from a dataset that can be identified for a particular purpose. ROI from image need to removefrom input image.

\subsection{Down sampling}

Down sapling is process of reducing a sampling rate (pixel size) of a signal.So apply down sampling on the ROI generated image.

\subsection{Training samples (Dictionary method)}

Training samples contain both input image and cropped image for in-painting.

\subsection{In-painting algorithm}

In-painting is the technique used for refill regions and recover the corrupted image.

\subsection{SR image}

Super resolution (SR) is a method of creating high resolution image from one or more low resolution image and generates the desired output.

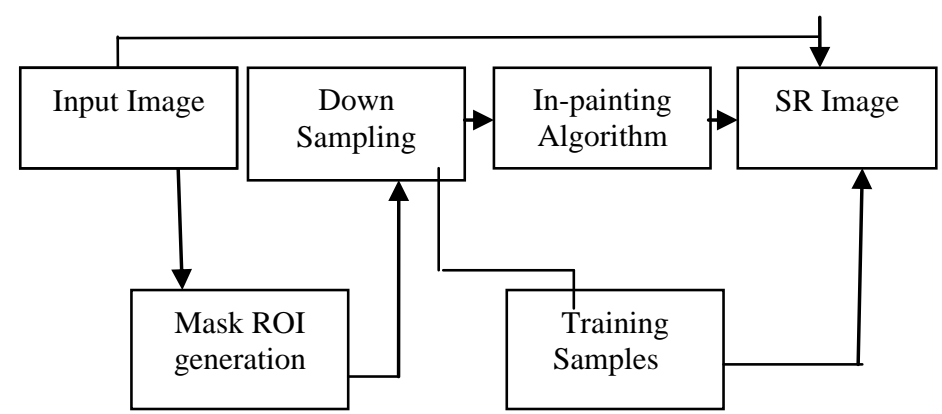

Fig.2System architecture of image in-painting

\section{PROPOSED ALGORITHM}

1. Firstly a low resolution image is build from a initial image.

2. An in-painting algorithm is applied to fill missing region of low resolution image.

3. The quality of the in-painted regions is improved after applying in-painting. Then the algorithm thus proceeds by searching for $\mathrm{K}$ nearest neighbors to the input vector concatenating the known HR (High- 
Resolution) pixels of the patch and the pixels of the corresponding in-painted LR patch.

Algorithm works as below.

Input:Original Image- It is an image which needs to be inpainted.

Mask Image- This image specifying the object to be removed or the regions to be inpainted. The user

marks the object to be removed with black color and other region with black color. Using this image

mark the object with red color in original image. This object which is marked in red is removed in

visual plausible way.

Output:Inpainted image- Output image include with the removal of object specify in mark image using inpainting

algorithm in visual plausible way.

Steps of algorithm are given below:

Step 1 : Initialize mark variable for all pixel. If pixel belongs to inpainting region set mark variablewith 0 else set 1

Step 2 : Find boundary of region to be inpaint, if boundary is empty set than exit.

Step 3 : Find priority for all patches on the boundary.

Step 4 : Select the patch which has highest priority, call that patch, P.

Step 5 :Find the patch from search window which is best match to patch $\mathrm{P}$, call that patch,Q.

Step 6 : Copy pixels of patch $\mathrm{Q}$ to the patch $\mathrm{P}$, update only those pixels of patch $\mathrm{P}$ which has mark

value 0 and set mark variable to1, go to step2.

\subsection{Extract targeted region}

From input image I,user selects a target region, as $\Omega$ to be filled and removed.The source region $\Phi=$ The entire image(I) minus the target region $(\Omega)$ like as $(\Phi=\mathrm{I}-\Omega), \Psi \mathrm{p}$ is the patchacross point $\mathrm{p}$ (say $9 \times 9$ pixel).

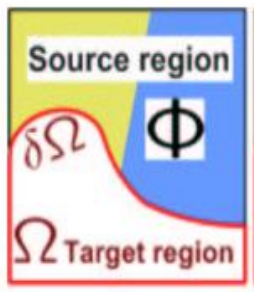

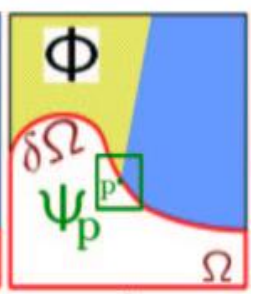

Fig.3.(a)Original image with target region $\Omega$, itscontour $\delta \Omega$ and the source region $\Phi(b)$ Synthesize the area determine by patch $\Psi p$ centered at the point $p \epsilon \delta \Omega$

\subsection{Computing patch priorities}

From a patch $\Psi p$ centered at point $p$, call its priority $\mathrm{P}(\mathrm{p})$ as the product of two terms $\mathrm{P}(\mathrm{p})=\mathrm{C}(\mathrm{p})$.

\subsection{Propagating texture and structure information}

Once all priorities computed, highest priority is found with the patch $\Psi \mathrm{p}$ then find in the source region for that patch which is most similar to patch $\Psi \mathrm{p}$. Distance between two generic patches is called sum of squared differences (SSD).

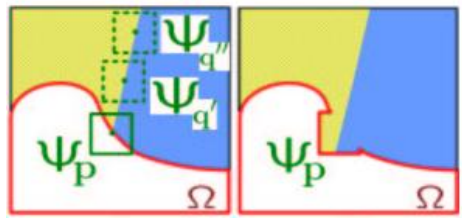

c

Fig.4 (c)The one of the most similar candidate matches for $\Psi p$ lie along the boundary between the two texture the source region (d)

Copy the best matching patch in the candidates set into the position occupied by $\Psi \mathrm{p}$, thus partial filling of $\Omega$ can be achieve.Space Complexity of In-painting:Space complexity S, of in-painting algorithm will be dependent on:Patch size ( $\mathrm{s}$ constant value), Input image size ( $\mathrm{X}$ m pixels, $\mathrm{N}=\mathrm{n} * \mathrm{~m}$ total pixels), Space needed for data dictionary $(\mathrm{d}=\mathrm{N}-\mathrm{s}) \mathrm{Hence}$ the total space needed is $\mathrm{S}=\mathrm{O}(\mathrm{N}+\mathrm{d})$

Time complexity $\mathrm{T}$, of in-painting algorithm will be dependent on:Input image size ( $\mathrm{n} X \mathrm{~m}$ pixels, $\mathrm{N}=\mathrm{n} * \mathrm{~m}$ total pixels), Number of Iteration (i) to perform action per pixel $(i * n)$,Size of data dictionary to search a particular patch (d)Hence the total time isT $=\mathrm{O}(\mathrm{i} * \mathrm{n} * \mathrm{~d})$

\section{EXPERIMENTAL SETUP AND EVALUATION}

In this section the experiment results on image is shown with four different stages(Fig. 5).First image is captured by camera, then, this image is given as the input to the preprocessing. In preprocessing it checks resolution of image. After preprocessing this will give input to the next stage. In next stage user need to select area which he want to in paint. Fig. 5(b) shows the region of interest. After selection of the object that selected object must remove from image,then using in painting algorithm the nearest patches should select to form the complete image Figure shows in (c).By using super resolution algorithm low resolution image is converted into high resolution image shows in Fig. 5(d).
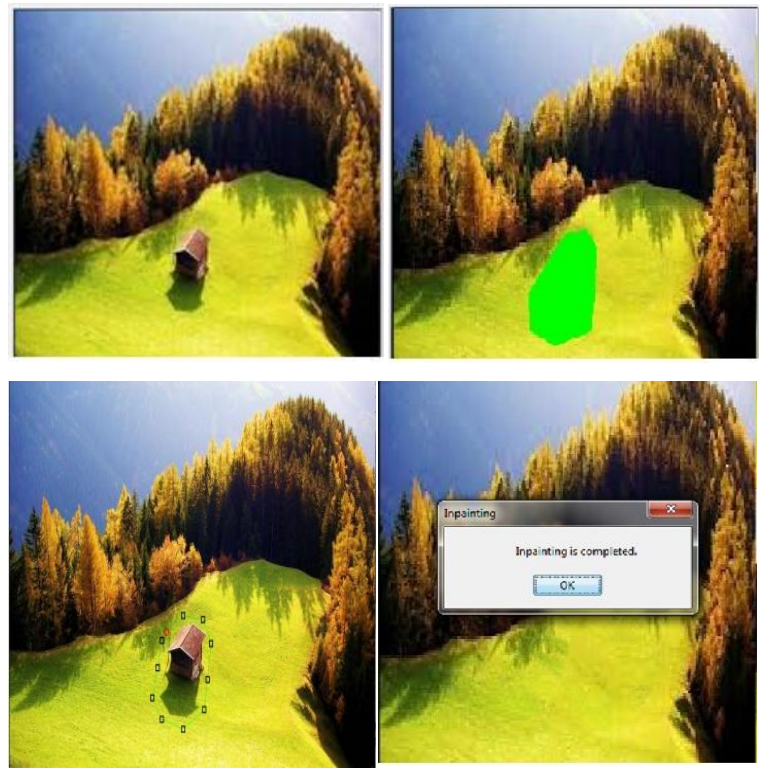


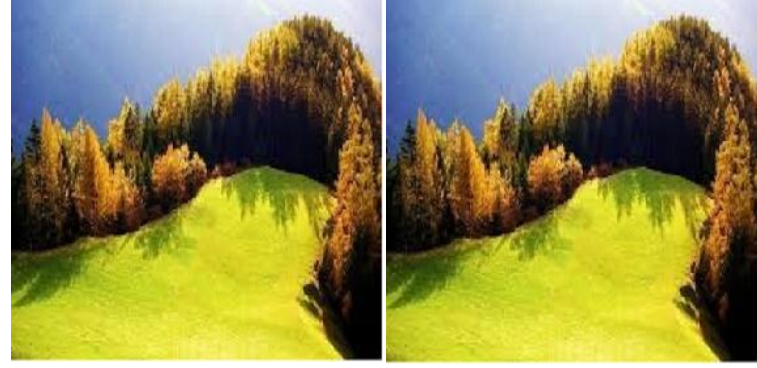

Fig. 5 Result of in painting.(a)Original image (b)Selected ROI (c) In painting procedure (d) Final result after applying super resolution algorithm

\section{CONCLUSION}

In this paper first review on existing methods of image in painting with their key ideas, advantages, disadvantages, applications. So there is lot of scope to work with the image in-painting. The attempt is to improve the performance in term of accuracy. The proposed method can be improved on exemplar based in-painting method by introducing new framework with combination of multiple in-painting versions of input image followed by single image exemplar based SR method. The future enhancement of this system is to conduct more experiments to examine the in-painting procedure with more algorithms and for specific applications. Real time in painting, super resolution can be used in various fields like Face or Number Plate detection in surveillance cameras. This technique can also be used to implement video in-painting for removing unwanted moving objects from the motion film.

\section{ACKNOWLEDGMENTS}

I thank Prof. R.V. Dagade for assistance with Image Inpaintingtechnique for comments that greatly improved the manuscript.

\section{REFERENCES}

[1] FaouziBenzarti, Hamid Amiri, TSIRF (ENIT)-TUNISIA (2012). "Repairing and In-painting Damaged Images using Diffusion Tensor" IJCSI International Journal of Computer Science Issues, Vol 9, Issue 4, No 3 , July 2012 ISSN 1694-0814.

[2] Jason C. Hung, Chun-Hong Hwang, Yi-Chun Liao, Nick C. Tangand Ta-Jen Chen, "Exemplar-based Image Inpainting base onStructure Construction", JOURNAL OF SOFTWARE, VOL. 3, NO. 8, NOVEMBER 2008.

[3] M. Bertalmio, L. Vese, G. Sapiro And S. Ocher, "Simultaneous

Structure and Texture Image In-painting" IEEE TRANSACTIONS ON IMAGE PROCESSING, VOL. 12,NO. 8, AUGUST 2003

[4] ShivaniGaikar, NehaKhairnar, Nikita Rane and M. J. Surti, "Image Inpainting using Exemplar based, DCT and FMM Algorithm",International Conference of Advance Research and Innovation, ISBN 978-93-5156-328-0, 2014.

[5] Manali Desai, "Modified Fast and Enhanced Exemplar based In painting Algorithm for Solving Unknown Row Filling Problem",International Journal of Computer
Applications (0975 - 8887, volume 56- No.9, October 2012.

[6] SheetalBadgujar and N. M. Shahane, "Examplar-based ImageInpainting and Approaches to Improve the Performance",International Journal of Computer Applications (0975 - 8887), 2013.

[7] Efros, A.A., Leung, T.K. "Texture synthesis by nonparametricsampling" IEEE International Conference on Computer Vision, Gorfu, Greece, September.

[8] G.AntoSilviya and V.R.Bhuma, "Exemplar-Based Image Inpainting by Laplacian Approximation Method Using Spatiogram”, International Journal of Engineering Research and Applications (IJERA) ISSN: 2248-9622.

[9] Criminisi, A., Prez, P., Toyama, K. Region filling and object removal by Exemplar-based image in-painting IEEE Trans. On Image Processing 2004.

[10] Olivier Le Meur and Christine Guillemot 'SuperResolution- based In-painting' IEEE TRANSACTIONS ON IMAGE PROCESSING VOL.PP NO 99 YEAR 2013.

[11] MouniraEbdelli, and Christine Guillemot'Hierarchical Super-Resolution-Based In-painting', IEEE TRANSACTIONS ON IMAGE PROCESSING, VOL. 22, NO. 10, OCTOBER 2013.

[12] S.Priyalakshmi, C.K.Sangeetha, S.Vijayalakshmi, T.C.Monica 'Super Resolution Image Reconstruction Using fast In-painting Algorithm', International Journal of Scientific \& Engineering Research, Volume 5, Issue 8,August-2014 1243 ISSN 2229- 5518.

[13] Manoj K Y , M r . Venkatesh U C,D r . A n tony P J 'A Superresolution Based Image In-painting in the Advertence of Exemplar Technique', International Journal of Engineering Research \& Technology (IJERT) IJERTIJERT ISSN: 2278-0181 Vol. 3 Issue 4, April - 2014.

[14] Glasner, D., Bagon, S., Irani, M.: Super-resolution from a single image. In: In 2009 IEEE 12th International Conference on omputer Vision (ICCV). Volume 10. (2009) .

[15] Sreelekshmi Das and Reeba R. Robust Exemplar based ObjectRemoval in Video International Journal of Scientific Engineering and Research (IJSER), ISSN (Online): 2347 - 3878, Volume 1 Issue 2, October 2013.

[16] Pratika Patel, AnkitPrajapati, Shailendra Mishra "Review of Different In-painting Algorithms",2012 International Journal of ComputerApplications.

[17] Anupam, PulkitGoyal, SapanDiwakar "Fast and Enhanced Algorithm for Exemplar Based Image Inpainting",

[18] A. J. Thesiya, D. U. Shah, P. V. Kathiriya, G. B. Vasani and J. L.Vyas "Image In-painting Realization of GUI in JAVA Using Exemplar Method", 2013IOSR Journal of Electronics and Communication Engineering (IOSRJECE). 